\title{
Anti-PD-1/TIM-3 Bispecific Antibody RO7121661
}

National Cancer Institute

\section{Source}

National Cancer Institute. Anti-PD-1/TIM-3 Bispecific Antibody R07121661. NCI

Thesaurus. Code C160714.

A bispecific antibody directed against both the negative immunoregulatory human cell receptor programmed cell death protein 1 (PD-1; PDCD1; CD279) and the inhibitory Tcell receptor T-cell immunog lobulin and mucin domain-containing protein 3 (TIM-3; TIM3; hepatitis A virus cellular receptor 2; HAVCR2), with potential immune checkpoint inhibitory and antineoplastic activities. Upon administration, anti-PD-1/TIM-3 bispecific antibody RO7121661 simultaneously targets and binds to both TIM-3 and PD-1 expressed on certain T-cells. This blocks the interaction of TIM-3 with some of its physiologic ligands and prevents the activation of PD-1 by its ligands, programmed cell death-1 ligand 1 (PD-L1) or 2 (PD-L2). This abrogates T-cell inhibition, activates antigenspecific T-lymphocytes and enhances cytotoxic T-cell-mediated tumor cell lysis, which may lead to a reduction in tumor growth. TIM-3, a transmembrane protein and immune checkpoint receptor, is often co-expressed with PD-1 on tumor-antigen-specific T-cells. Dual checkpoint blockade of PD-1 and TIM-3 may enhance T-cell activation and proliferation more than the blockade of either immune checkpoint receptor alone. 